\title{
Are the boys back in town? Zur anhaltenden Bedeutung realistischer Theorien
}

Die jüngsten Ereignisse in Europa haben viele politische und politikwissenschaftliche Beobachter irritiert. Kaum jemand hätte erwartet, dass die Russische Föderation unter Einsatz von militärischen Machtmitteln einen Teil eines souveränen Staates annektiert (die Krim) sowie aktiv die Destabilisierung der gesamten Ukraine betreibt. Unabhängig von der politischen und völkerrechtlichen Einschätzung des russischen Verhaltens ist auch die Tatsache, dass der aktive und passive Gebrauch militärischer Machtmittel, das Denken in geostrategischen Kategorien nach Europa zurückgekehrt sind, für viele Beobachter überraschend. Dabei zeigt die Krise in und um die Ukraine doch wieder einmal nur sehr deutlich, dass Realpolitik in der internationalen Politik weiterhin eine zentrale Strategie von Staaten ist (und bleibt) und dass realistische Theorien Internationaler Politik (in ihren diversen Spielarten) zur Erklärung solcher Politiken weiterhin Relevanz beanspruchen können.

Seit nunmehr fast fünfundzwanzig Jahren versuchen führende und weniger führende Kolleginnen und Kollegen der politikwissenschaftlichen Teildisziplin Internationale Politik den Studierenden sowie ihren Fachkollegen darzulegen, dass militärische Macht, Denken in Kategorien des nationalen Interesses sowie der Geostrategie oder der Geopolitik Relikte des 18. oder 19. Jahrhunderts sind. Die „moderne" Internationale Politik, so ihr Narrativ, sei von wirtschaftlichen Interessen und globalen Problemen gekennzeichnet, die Staaten, sei es in internationalen Organisationen oder in diversen Formen von Global Governance, gewissermaßen zur Kooperation zwingen würden (Weiss/Wilkinson 2014). Militärische Macht, so der Tenor, sei heute kein bestimmender Faktor der Beziehungen zwischen Staaten (Schweller 2014: 85-88). Diese Einschätzung wird auch von der operativen Politik geteilt. U.S.- Außenminister John Kerry (zitiert nach Mearsheimer 2014: 84) kleidete seine Fassungslosigkeit über das russische Vorgehen auf der Krim in die Worte: "You just don't in the twenty-first century behave in nineteenth-century fashion by invading another country on completely trumped-up pretext."

Wissenschaftler, die auf der Basis realistischer Theorien arbeiten, waren von diesen Entwicklungen weniger überrascht, und zwar nicht aufgrund detaillierter Analysen der russischen Politik, sondern aufgrund ihrer grundsätzlichen Einschätzung 
der Hauptantriebskräfte internationaler Politik. Realistisch arbeitende Autoren ${ }^{1}$ gehen in ihren Analysen von drei zentralen Annahmen aus:

a) Gruppen. Politik findet in und zwischen Gruppen (sozialer Art) statt. Gruppensolidarität ist zentral für innenpolitische Prozesse und Konflikte sowie Kooperation zwischen Gruppen. Sie ist der Kern internationaler Politik.

b) Egoismus. In ihrem Handeln sind Individuen und Gruppen im Wesentlichen durch ihre eigenen Interessen getrieben.

c) Machtzentrismus. Der Schlüssel zum Verständnis von Politik (sei es Innen-, Außen- oder internationale Politik) ist die Interaktion von sozialer und materieller Macht. Diese Interaktion findet beständig vor dem Hintergrund eines möglichen Gebrauchs materieller Macht zum Zwecke des Zwangs statt (Wohlforth 2010: 9-20).

Connaisseurs realistischer Theorien werden an dieser Stelle bemerken, dass Annahmen über die Struktur des internationalen Systems nicht zum gemeinsamen Kanon realistischer Theorien gehören. Dies hat insofern seine Berechtigung, als strukturell arbeitende Realisten (Waltz 1979) nur einen Teil der realistischen Familie ausmachen, auch wenn dieser Teil in den 1980er und 1990er Jahren einen prominenten Stellenwert innerhalb des realistischen Theorienkanons eingenommen hat.

Ausgehend von diesen Annahmen argumentieren (fast) alle Realisten, dass Staaten in der internationalen Politik die wichtigsten Akteure sind, deren primäres Interesse ihrem Überleben gilt. Das Interesse am Überleben hat für Staaten gegenüber allen anderen möglichen Zielen eine quasi „lexikalische Priorität“"(Rawls 1971: 63). Wenn dieses Überleben, verstanden als Überleben des Regimes als autonomer Akteur auf seinem Territorium, auf dem Spiel steht oder befürchtet wird, dass es auf dem Spiel stehen könnte, dann sind soziale Akteure bereit, zu allen ihnen zur Verfügung stehenden Mitteln zu greifen - inklusive der Androhung und des Einsatzes militärischer Gewalt -, um dieses Überleben zu garantieren (Monteiro 2014: 32). Ist das Überleben aus Sicht der Akteure garantiert oder zumindest nicht akut bedroht, dann können und werden sie andere Prioritäten (z.B. ökonomischen Wohlstand) verfolgen. Aber: Es bleibt immer die Ungewissheit über die (wahren) Intentionen von Staaten bestehen (Mearsheimer 2001), die staatliche Akteure beständig um ihr Überleben bangen lässt.

Will man mit diesen Annahmen das gegenwärtige russische Verhalten erklären, so ist es - kurz gesagt - der Dreiklang aus NATO- und EU-Erweiterung sowie die

1 Ich selbst habe an verschiedenen Stellen (siehe insbesondere 2015) dargelegt, dass es den Realismus als einheitliche Theorie der Internationalen Politik nicht gibt. Vielmehr gibt es divergierende, sich ergänzende und miteinander konkurrierende Theorien, die (fast) alle auf einem gemeinsamen Set an Annahmen beruhen. 
von westlichen Staaten und Institutionen aktiv betriebene Demokratisierungspolitik (die Russlands Vorfeld transformieren soll und die Russland selbst seit geraumer Zeit an den Pranger stellt), der bei den politischen und militärischen Eliten in Moskau die Alarmglocken schrillen lässt und die Befürchtung eines Verlusts der Autonomie - im Sinne der Freiheit der gesellschaftlichen Eigenentwicklung - sowie Einkreisungsängste schürt. Auf diese Entwicklungen und die daraus resultierenden Befürchtungen haben Putin und sein Umfeld reagiert.

Aber auch über das russische Verhaltens gegenüber der Ukraine hinaus wird seit Jahren immer deutlicher, dass es in den 1990er Jahren verfrüht war, das Sterbeglöckchen für realistische Theorien zu läuten. Denn die internationale Politik wird zunehmend von der Frage des Aufstiegs neuer Mächte und der Auswirkung, die dieser Aufstieg auf die ,alte“ internationale Ordnung hat, bestimmt. Fragen von Macht und Gegenmacht sowie der daraus möglicherweise resultierenden Konfliktanfälligkeit des internationalen Systems beschäftigen die Politik zusehends. Und auch Wissenschaftler, die bislang eher als Vertreter liberaler Theorien galten, entdecken bei der Analyse dieser Frage realistische Konzepte wie zum Beispiel das Konzert der Mächte (Müller u. a. 2014), Eindämmung (Nye 2008: 652) oder das realistische „Tafelsilber“ des Macht- und Sicherheitsdilemmas (Herz 1950), welches zwischen den aufsteigenden Mächten des 21. Jahrhunderts sowie den USA Wirkungsmacht entfaltet (Drezner 2008).

Am auffälligsten ist die Renaissance realistischer Theorien bei der Diskussion um die Folgen des chinesischen Aufstiegs. Diese Diskussion findet allerdings weniger unter deutschen oder europäischen, sondern vor allem unter U.S.-amerikanischen Akademikern statt. Kaum ein Beobachter des chinesischen Aufstiegs und seiner möglichen Folgen für die internationale Politik der Zukunft negiert, dass dieser Aufstieg der rasanten internen Machtsteigerung Chinas (sowohl im ökonomischen wie auch im militärischen Bereich) entspringt und das Potenzial hat, die zukünftige Struktur des internationalen Systems mit Blick auf dessen Polarität zu verändern. Sicher bildet John Mearsheimer mit seiner Prognose zum bevorstehenden Krieg zwischen den USA und China die (wie so oft) extreme Ausnahme, wenn es darum geht, über die Folgen des chinesischen Aufstieges nachzudenken, aber die Annahme, dass dieser Aufstieg eine enorme machtpolitische Herausforderung, insbesondere für die USA, darstellt, wird eigentlich von allen, und damit auch von nicht realistisch arbeitenden Wissenschaftlern, geteilt (für zwei Beispiele siehe Kupchan 2012: 102; Ikenberry 2008: 24). Die Idee, dass überproportionale Machtzuwächse eines Staates bei anderen Staaten Sicherheitsbedenken produzieren, gehört aber zum Kern (fast) aller realistischen Theorien (Jervis 1978). 
Diese drei - zugegebenermaßen holzschnittartig - skizzierten Beispiele verdeutlichen zwei Tendenzen. Zum ersten, realistische Theorien haben nach wie vor einen prominenten Platz im Kanon der Theorien internationaler Politik. Sie können, insbesondere wenn es um das Verhalten von Großmächten geht oder wenn strukturelle Fragen der internationalen Politik im Vordergrund stehen, einiges besser erklären als konkurrierende Theoreme und wichtige Hinweise darauf geben, welche Entwicklungen bei der gegenwärtig stattfindenden Formierung des internationalen Systems zu erwarten sein könnten. Die Art und Weise, wie sich in der jüngsten Vergangenheit Nicht-Realisten realistischer Theoreme bedienen, zeigt, dass die Zeit der Neo-Neo-Debatte (die allgemeinhin als die zweite große Debatte in den IB bezeichnet wird, obgleich es nie eine echte Debatte, sondern eher ein Aneinandervorbeireden war), endgültig vorbei ist. Anstatt der Frage nachzulaufen, welche Theorie besser erklärt bzw. versteht, konzentrieren sich Autoren zunehemd darauf, die bestmöglichste Antwort auf eine Forschungsfrage zu finden. Und wenn dies bedeutet, dass realistische Variablen zur Erklärung einer bestimmten Frage mitberücksichtigt oder in die Theorie mitintegriert werden müssen, dann geschieht das eben: auf eine pragmatische Art und Weise. Theoriepuristen mag ein solches Vorgehen zuwider sein; so lange es uns aber in der Erkenntnis weiterbringt, ist dieser Eklektizismus gerechtfertigt.

Doch auch auf dem Gebiet der normativen Theorie gibt es, zweitens, Erstaunliches zu vermelden, was durchaus als „good news for realism“ bezeichnet werden kann. Zum einen bringen sich realistische Wissenschaftler immer stärker in die politische Debatte (insbesondere in den USA und bezogen auf die gegenwärtige und zukünftige Außen- und Sicherheitspolitik) ein. Die verschiedenen Überlegungen, die in diesem Zusammenhang angestellt wurden, lassen sich alle unter dem Begriff „Kultur der Zurückhaltung“ (Posen 2014) zusammenfassen. Stephen Walt (2005) etwa empfiehlt den USA, sich weitestgehend aus Konflikten in Übersee herauszuhalten und nur dann aktiv einzugreifen, wenn die nationalen Interessen der USA gefährdet sind. Eine Fortführung des globalen Engagements der USA würde, auch wenn deren Intentionen gut gemeint seien, ,alarm, irritate, and at times anger others“ (Walt 2005: 60). John Mearsheimer (2001) und Christopher Layne (2002) empfehlen den USA, sich auf die Strategie des offshore-balancing zu beschränken. Diese sieht im Kern vor, dass die Vereinigten Staaten nur dann aktiv werden, wenn sich irgendwo auf der Welt eine Situation abzeichnet, in der ein anderer Staat oder eine Staatengruppe sich anschickt, die regionale Hegemonie über eine Landmasse zu erlangen. Die aus der Perspektive von John Mearsheimer und Stephen Walt traditionelle ,grand strategy“ (2008: 339) der USA ist die einzige Möglichkeit, so Dale 
Copeland (2000), Gegenmachtbildung gegenüber den Vereinigten Staaten zu vermeiden bzw. noch über einen gewissen Zeitraum hinauszuzögern.

Das Reden und Schreiben in der Agora ist insofern erstaunlich, als Realisten normalerweise der Auffassung sind, dass der „Marktplatz der Ideen“ (Kaufmann 2004) keinerlei Bedeutung für und keinerlei Einfluss auf politische Entscheider hat. John Mearsheimer selbst ist es, der Hinweise darauf gibt, dass sein Engagement von starken ethischen und normativen Beweggründen geleitet wird, die unter dem Stichwort der sozialen Verantwortung der Politikwissenschaft gegenüber der Gesellschaft subsumiert werden können. Dabei - und dies ist in diesem Zusammenhang von besonderem Interesse - stellt er sein politisches Engagement in einen direkten Zusammenhang mit seinem theoretischen Denken und begibt sich dadurch selbst in einen Widerspruch zu der immer wieder in seinen Schriften vorzufindenden Skepsis gegenüber einem möglichen Einfluss der Agora auf die Politik (Mearsheimer 2002: 4-7).

Es ist beileibe nicht so, dass dieser Beitrag die Rückkehr realistischer Theorien als das alte/neue Primat der Internationalen Beziehungen lobpreisen will, er will jedoch darauf aufmerksam machen, dass realistische Theorien auch heute noch einige wichtige Faktoren in der realen internationalen Politik besser erklären können als ihre Hauptkonkurrenten. Und dass viele realistische Konzepte beileibe nicht obsolet sein können, wie seit nunmehr zwanzig Jahren suggeriert wird, wenn sie angesichts aktueller politischer Entwicklungen vermehrt zur Analyse (insbesondere von Nicht-Realisten) herangezogen werden. Für Realisten ist dies beileibe kein Anlass für triumphierendes Geheul, denn es sollte sie nicht davon entlasten, den Dialog mit anderen Theorien (Hellmann 2002) zu suchen.

Eine Reihe von Gemeinsamkeiten sieht der Verfasser des vorliegenden Essays mit post-strukturalistischen Theorien (und an dieser Stelle werden Vertreter dieser Richtung sicher aufschreien) und zwar auf der Ebene der normativen Analyse internationaler Politik. Mögliche ontologische und epistemologische Barrieren dürfen dabei getrost ignoriert werden. Interessanterweise treffen sich postmoderne und realistische Diskurse nämlich dort, wo es um normative Empfehlungen an die reale Politik geht. Chantal Mouffe zum Beispiel fordert die Schaffung einer multipolaren Weltordnung, um Übermacht auszubalancieren. , [T] he only conceivable strategy”, so schreibt sie, ,for overcoming world dependence on a single power is to find ways to 'pluralize' hegemony" (Mouffe 2005: 118). Und Danilo Zolo, ein postmoderner Philosoph aus Italien, spricht sich zum Zwecke der Einhegung US-amerikanischer Übermacht im 21. Jahrhundert für die Schaffung regionaler Blöcke aus (Zolo 2002: 85). Unlängst hat Giorgio Agamben die Schaffung eines lateinischen Imperiums in Europa unter französischer Führung vorgeschlagen, um die ökonomische und po- 
litische Hegemonie Deutschlands in der EU auszutarieren (Agamben 2013). All dies klingt wie viele Policy-Empfehlungen, die realistisch arbeitende Autoren in der Vergangenheit gegeben haben (Waltz 2000). Zur Überraschung einiger Fachkollegen hat Peter Gowan, ein britischer marxistischer Historiker, unlängst angemerkt, dass die Linke von John Mearsheimers „Tragedy of Great Power Politics” mehr lernen könne ,than from any number of treaties from the coming wonders of global governance" (Gowan 2002: 67).

Zunächst einmal erscheinen problemlösungsorientierte und kritische Theorie, so die berühmte Unterscheidung von Robert Cox (1981), als seltsame Bettgefährten. Doch ein genauer Blick, insbesondere wenn es um Empfehlungen an die praktische Politik geht, zeigt, dass beiden die Geisteshaltung gemeinsam ist, etablierte Ordnungen kritisch zu hinterfragen trotz des grundlegenden Unterschiedes, dass Realisten skeptisch hinsichtlich der Möglichkeit von , human emancipation“(Cox 1996: 53) sind. Realisten sollten künftig akzeptieren, dass ihre Theorien neben einer analytischen auch eine normative Dimension haben (Masala 2011, 2014: 161-171). Wird diese doppelte Dimension realistischer Theorie von Realisten akzeptiert, öffnet sich die diskursive Tür für einen fruchtbaren Dialog mit postpositivistischen Theorieansätzen. Seitens der Postpositivisten sollte aber auch die Einsicht greifen, dass eine positivistische Grundhaltung in Wissenschaftsfragen kein Hindernis ist, um sich an öffentlichen Diskursen zu beteiligen und dazu auch nicht im Widerspruch steht.

Eine weitere wichtige Aufgabe neorealistisch argumentierender Forschung besteht - gerade in der heutigen Zeit, in der der Neorealismus seine Rolle als führendes Paradigma der Disziplin Internationale Beziehungen eingebüßt hat und nicht selten als degeneriert betrachtet wird - darin, die Rolle der ,skeptischen Gegenwartswissenschaft“ ${ }^{\text {‘ }}$ einzunehmen. Denn in Zeiten, in denen konstruktivistischen Arbeiten und andere dem Post-Positivismus verpflichteten Ansätzen die Rolle einer neuen Orthodoxie an Universitäten zufällt, bedarf es Forschern, die deren Ergebnisse kritisch hinterfragen. Für manch etablierten Neorealisten mag die Vorstellung des Neorealismus als einer „skeptischen Gegenwartswissenschaft“ nur schwer mit ihrem eigenen Verständnis vom Neorealismus als der einzig wahren Theorie in Einklang zu bringen sein. Nichtsdestotrotz liegt letzten Endes hier die Zukunft neorealistischer Theorie im 21. Jahrhundert. Trotz all der Hausaufgaben, die realistische Theoretiker noch zu machen haben, gilt fraglos Thin Lizzy's Motto: „The boys are back in town".

2 Diese Anregung verdanke ich Jürgen Neyer. 
Are the boys back in town? Zur anhaltenden Bedeutung realistischer Theorien

\section{Literatur}

Agamben, Giorgio, 2013: Se un impero latino prendesse forma nel cuore d'Europa, la Repubblica.it, 15.3.2013, http://ricerca.repubblica.it/repubblica/archivio/repubblica/2013/03/15/se-un-impero-latino-prendesse-forma-nel.html (Stand: 22.3.2014).

Copeland, Dale C., 2000: The Origins of Major War, Ithaca, NY.

Cox, Robert, 1981: Social Forces, States and World Orders: Beyond International Relations Theory, in: Millennium - Journal of International Studies 10 (2): 126-155.

Cox, Robert W., 1996: Realism, Positivism, and Historicism, in: Robert W. Cox/ Timothy J. Sinclair (Hrsg.), Approaches to World Order, Cambridge, 49-59.

Drezner, Daniel, 2008: BRIC by BRIC: The emergent regime for sovereign wealth fund, Prepared for the Princeton summer workshop on Rising States and Rising Institutions, http://danieldrezner.com/research/swf1.pdf (Stand: 31.8.2014).

Gowan, Peter, 2002: A Calculus of Power, in: New Left Review 16, 47-67.

Hellmann, Gunther, 2002: Creative Intelligence. Pragmatism as a Theory of

Thought and Action (Paper prepared for presentation at the "Millennium" Special Issue Conference on "Pragmatism in International Relations Theory", London, 12 October 2002), London.

Herz, John H., 1950: Idealist Internationalism and the Security Dilemma, in: World Politics 2 (2), 157-180.

Ikenberry, G. John, 2008: The rise of China and the future of the West: Can the liberal system survive? Foreign Affairs 87 (1), 23-37.

Jervis, Robert, 1978: Cooperation under the Security Dilemma, in: World Politics 30 (2), 167-174.

Kaufmann, Chaim, 2004: Threat Inflation and the Failure of the Marketplace of Ideas: The Selling of the Iraq War, in: International Security 29 (1), 5-48.

Kupchan, Charles A., 2012: No one's world. The west, the rising rest, and the coming global turn, Oxford.

Layne, Christopher, 2002: Offshore Balancing Revisited, in: The Washington Quarterly 25 (2), 233-248.

Masala, Carlo, 2011: Warum (Neo-)Realisten (meistens) keinen Krieg mögen, in: Zeitschrift für Außen- und Sicherheitspolitik 4, 253-269.

Masala, Carlo, 2014: Kenneth N. Waltz. Einführung in seine Theorie und Auseinandersetzung mit seinen Kritikern, 2., vollständig überarbeitete und erweiterte Auflage, Baden-Baden. 
Masala, Carlo, 2015: Realismus, in: Carlo Masala/Frank Sauer (Hrsg.), Handbuch der Internationalen Beziehungen, 2., vollständig überarbeitete und erweiterte Auflage, Wiesbaden (i. E.).

Mearsheimer, John J., 2001: The Tragedy of Great Power Politics, New York, NY. Mearsheimer, John J., 2002: Liberalist Talk, Realist Thinking, in: University of Chicago Magazine 94 (3), 1-7.

Mearsheimer, John J./Walt, Stephen M., 2008: The Israel Lobby and U.S. Foreign Policy, New York, NY.

Mearsheimer, John J., 2014: Why the Ukrainian crisis is the West's fault. The liberal delusions that provoked Putin, in: Foreign Affairs 93 (5), 77-89.

Mouffe, Chantal, 2005: On the Political, New York, NY.

Müller, Harald/Jüngling, Konstanze/Müller, Daniel/Rauch, Carsten, 2014: Ein Mächtekonzert für das 21. Jahrhundert, HSFK-Report Nr. 1/2014, Frankfurt a. M.

Monteiro, Nuno P., 2014: Theory of Unipolar Politics, Cambridge, MA.

Nye, Joseph, 2008: International Relations: The Relevance of Theory to Practice, in: Christian Reus-Smit/Duncan Snidal (Hrsg.), The Oxford Handbook of International Relations, Oxford, 648-662.

Posen, Barry R., 2014: Restraint. A New Foundation for U.S. Grand Strategy, Ithaca, NY.

Rawls, John, 1971: A Theory of Justice, Cambridge, MA.

Schweller, Randall L., 2014: Maxwell's Demon and the Golden Apples. Global Disorder in the New Millennium, Baltimore, MD.

Walt, Stephen M., 2005: Taming American Power: The Global Response to U.S. Primacy, New York, NY.

Waltz, Kenneth N., 1979: Theory of International Politics, Reading, MA.

Waltz, Kenneth N., 2000: Structural Realism after the Cold War, in: International Security 25 (1), 5-41.

Weiss, Thomas G./Wilkinson, Rorden, 2014: Global Governance to the Rescue:

Saving International Relations?, in: Global Governance: A Review of Multilateralism and International Organizations 20 (1), 19-36.

Wohlforth, William C., 2010: Realism and Security Studies, in: Myriam Dunn Cavelty/Victor Mauer (Hrsg.), The Routledge Handbook of Security Studies, New York, NY, 9-20.

Zolo, Danilo, 2002: Invoking Humanity: War, Law and Global Order, London. 
Korrespondenzanschrift:

Prof. Dr. Carlo Masala

Institut für Politikwissenschaft

Staats- und Sozialwissenschaftliche Fakultät

Universität der Bundeswehr München

Werner-Heisenberg-Weg 39

85577 Neubiberg

E-Mail: carlo.masala@unibw.de 